\title{
Genetic and Non-Genetic Factors Impact on INR Normalization in Preprocedural Warfarin Management
}

\author{
Islam Eljilany, (D) \\ Mohamed Elarref, ${ }^{2}$ \\ Nabil Shallik, (D) ${ }^{2-5}$ \\ Abdel-Naser Elzouki, (iD) ${ }^{6-8}$ \\ Loulia Bader,' Ahmed El-Bardissy, ${ }^{9}$ \\ Osama Abdelsamad, ${ }^{10}$ Daoud \\ Al-Badriyeh,' Larisa H Cavallari,' \\ Hazem Elewa (1) 1, 10,12 \\ 'College of Pharmacy, QU Health, Qatar \\ University, Doha, Qatar; ${ }^{2}$ Department of \\ Anesthesia, Hamad General Hospital, \\ Hamad Medical Corporation, Doha, Qatar; \\ ${ }^{3}$ Department of Anesthesia, Faculty of \\ Medicine, Tanta University, Tanta, Egypt; \\ ${ }^{4}$ Department of Clinical Anesthesia, College \\ of Medicine, Qatar University, Doha, Qatar; \\ ${ }^{5}$ Department of Clinical Anesthesia, Weill \\ Cornell Medical College, Doha, Qatar; \\ ${ }^{6}$ Department of Medicine, Hamad General \\ Hospital, Hamad Medical Corporation, \\ Doha, Qatar; ${ }^{7}$ Department of Internal \\ Medicine, College of Medicine, Qatar \\ University, Doha, Qatar; ${ }^{8}$ Department of \\ Internal Medicine, Weill Cornell Medical \\ College, Doha, Qatar; ${ }^{9}$ Department of \\ Pharmacy, Hamad General Hospital, Hamad \\ Medical Corporation, Doha, Qatar; \\ ${ }^{10}$ Department of Pharmacy, Al Wakra \\ Hospital, Hamad Medical Corporation, \\ Doha, Qatar; "Department of \\ Pharmacotherapy and Translation Research, \\ Center for Pharmacogenomics and \\ Precision Medicine, College of Pharmacy, \\ University of Florida, Gainesville, FL, USA; \\ ${ }^{12}$ Biomedical and Pharmaceutical Research \\ Unit, QU Health, Qatar University, Doha, \\ Qatar
}

Correspondence: Hazem Elewa Biomedical and Pharmaceutical Research Unit, QU Health, Qatar University,

P.O. Box 2713, Doha, Qatar

Tel +97466628838

Fax +974 4403555 I

Email Hazem.elewa@qu.edu.qa
Background: Annually, $10 \%$ of warfarin patients will likely need to stop warfarin prior to elective surgery to achieve a baseline international normalization ratio (INR) level (INR $\leq$ 1.2) at the time of the procedure. This study explores the influence of genetic and nongenetic factors on INR normalization in the Arab (major part of Near Eastern) population in preprocedural warfarin management.

Methods: An observational prospective cohort study was designed to recruit Arab patients taking warfarin and scheduled for an elective procedure. Two INR readings were recorded. DNA extraction and genotyping of variants in $C Y P 2 C 9 * 2, C Y P 2 C 9 * 3, C Y P 4 F 2 * 3$, VKORC1*2, and FII (rs5896) and FVII (rs3093229) genes using real-time polymerase chain reaction were performed.

Results: Data from 116 patients were included in the analysis. CYP2C9 and VKORC1 genetic variants carriers required lower maintenance dose compared to non-carriers. The analysis showed that ciprofloxacin, antiplatelet medications, and INR index (INR at visit 1) are the only factors associated with the INR decline rate. Also, the proportion of $C Y P 2 C 9 * 3$ carriers with normal INR $(\leq 1.2)$ on the day of surgery was significantly lower than those with wild-type genotype ( $28 \%$ vs $60 \%, p=0.013$ ). In addition, heparin bridging, INR target, and Sudanese nationality are significant predictors of INR normalization $(\leq 1.2)$ on the day of the procedure.

Conclusion: Despite the confirmed effect of genetic factors on warfarin maintenance dose, the study was not able to find a significant effect of any genetic factor on the rate of INR normalization possibly due to the small sample size. Index INR and interacting medications showed to be significant predictors of INR decline rate.

Keywords: INR, periprocedural, pharmacogenetics, pharmacogenomics, warfarin

\section{Introduction}

Warfarin has been the mainstay oral anticoagulant (OAC) prescribed in thromboembolic conditions for several decades. ${ }^{1,2}$ While direct oral anticoagulants (DOACs) have been widely used lately, warfarin remains a very important option, especially in patients who cannot afford DOACs or have a contraindication to their use. ${ }^{3}$ With warfarin challenging narrow therapeutic index, the international normalized ratio (INR) is a handy surrogate marker to monitor warfarin's therapeutic effect. ${ }^{4}$ In procedures associated with more than minimal bleeding risk, warfarin-receiving patients are expected to stop it 5-7 days before surgery to achieve a baseline INR level and reduce the risk of bleeding during and after the procedure. ${ }^{5}$ However, the rate of INR decline varies across patients and may require a shorter or longer 
warfarin interruption period. Recent studies ${ }^{6,7}$ found that $23 \%$ of the patients who stopped warfarin still had an INR $>1.2$ following 4.7 days of warfarin holding, and $7 \%$ also had a preoperative INR $>1.5$ after 5 days. Furthermore, Spyropoulos et $\mathrm{al}^{8}$ and Pengo et $\mathrm{al}^{9}$ found that the mean \pm standard deviation (SD) INR was $1.8 \pm 0.5$ after 5-6 days of warfarin discontinuation.

Mechanistically, warfarin's elimination half-life plays a vital role in INR normalization rate and may at least partly explain this variability. ${ }^{10}$ Warfarin is a mixture of two active enantiomers: the (R) and (S) enantiomers, where the latter has five-fold higher anticoagulation potency than the former. ${ }^{11}$ Indeed, the S-enantiomer is metabolized by the Cytochrome P450-family 2, subfamily C, member 9 (CYP2C9) enzyme. ${ }^{12-14}$ Genetic variants in $C Y P 2 C 9$ have been widely studied, and it was found that $C Y P 2 C 9 * 2$ (rs1799853) and CYP2C9*3 (rs1057910) alleles are the most common variant alleles across majority of populations including the Middle Eastern and North Africa (MENA) population. ${ }^{15}$ These variants $(C Y P 2 C 9 * 2$ and $C Y P 2 C 9 * 3)$ account for reductions in the CYP2C9 enzymatic activity to $12-70 \%$ and almost no enzymatic activity, respectively. ${ }^{16}$ The Cytochrome P450-family 4, subfamily F, member 2 (CYP4F2) is another liver enzyme that inactivates the hydroxy-vitamin K1 and may also have an impact on warfarin and the rate of INR normalization. ${ }^{4}$ CYP $4 F 2 * 3$ (rs2108622) is a non-synonymous variant in the CYP4F2 gene that has been associated with a reduction in the CYP4F2 enzyme activity by $8-11 \%$ and may be associated with warfarin dose increase by $4-12 \%{ }^{5,17} \mathrm{CYP}^{2} \mathrm{~F} 2 * 3$ is a commonly occurring mutation in various populations and has a minor allele frequency (MAF) of $30-42 \%$ in the MENA region. ${ }^{18}$ The most crucial pharmacodynamic target for warfarin is vitamin $\mathrm{K}$ epoxide reductase complex subunit 1 (VKORC1) enzyme, which is inhibited by warfarin leading to reduction in the reduced form of vitamin $\mathrm{K}$ needed for the activation of its dependent coagulation factors (II, VII, IX, and $\mathrm{X}){ }^{19}$ The most common genetic variation in the VKORC1 gene is VKORC1*2 $-1639 \mathrm{G}>\mathrm{A}$ (rs9923231), which is relatively common (30-72\%) in patients of MENA descent. ${ }^{14,18}$ A recent local study showed that VKORC1 and CYP2C9 variants along with clinical factors can explain about $40 \%$ of warfarin dose variability in Qatari population. ${ }^{20}$ The update of Clinical Pharmacogenetics Implementation Consortium (CPIC) guideline in 2016 incorporated recommendations for warfarin dose based on the genetic information of CYP2C9, VKORC1 and CYP4F2 polymorphism. $^{21}$
Other than the pharmacokinetic and pharmacodynamic targets for warfarin, coagulation factors regeneration is considered a limiting step in INR decline rate; these factors' genetic polymorphisms may affect their regeneration and synthesis rates and eventually affect the time for INR normalization. ${ }^{10,22}$ The most common genetic polymorphisms in the gene encoding for factor VII (FVII) is (rs3093229), which has an average prevalence of $22 \%$ among diverse populations. ${ }^{23,24}$ Additionally, it has been found that patients' sensitivity to warfarin is generally affected by variations in factor II (FII) gene (rs5896). ${ }^{25,26}$ FII rs5896 variant has a prevalence of $14 \%$ among the American population and $13 \%$ among the Italians. ${ }^{25-27}$

Genetic factors that influence INR normalization during preprocedural warfarin interruption have been explored directly and indirectly in previous studies with various ethnic groups. ${ }^{28-34}$ Some found that CYP2C9 is the only genetic player, ${ }^{28,32,34}$ while others failed to find any association between genetic variants and INR decline. . $^{29-31,33}$

To the best of our knowledge, no studies investigated the effect of CYP2C9, CYP4F2, and VKORC1 genetic polymorphism on INR decline in the Arab (major part of Near Eastern) population. Moreover, coagulations FII and FVII polymorphisms on INR normalization have not been explored before. This study explored the influence of CYP2C9, VKORC1, CYP4F2, FII, and FVII genetic polymorphisms and non-genetic factors on INR normalization in the Arab (major part of Near Eastern) population in preprocedural warfarin management.

\section{Methods}

\section{Study Design and Ethics}

This study was designed as an observational prospective cohort study. Ethical approval was obtained from the Institutional Review Board (IRB) of Qatar University (QU) (QU-IRB 1296-FBA/20) and Hamad Medical Corporation (HMC) (MRC-16415/16). This study was conducted in accordance with the Declaration of Helsinki. Patient's informed consent was requested.

\section{Study Setting and Timeline}

The recruitment started in September 2018 and ended in December 2020. Afterward, genetic and data analyses were conducted in January 2021. Participants were recruited from the anesthesia, anticoagulation, and surgery clinics at Al Wakrah Hospital, Hamad General Hospital, 
Rumailah Hospital and Heart Hospital, which are all part of HMC, the largest healthcare corporation in Qatar.

\section{Study Population and Enrolment}

The study screened warfarin patients of the Arab population (being of any of the League of the Arab States). ${ }^{35}$ The patients reported their nationality verbally, and it was confirmed through the patient's electronic health record (EHR).

Subjects were eligible if they were $\geq 18$ years old, on warfarin for $\geq 1$ month with a stable INR for the last two consecutive visits with a minimum one-week interval (a stable INR was defined as INR within \pm 0.2 units of the target therapeutic range), ${ }^{36}$ undergoing elective surgery that required warfarin discontinuation for $\geq 3$ days. Exclusion criteria were patients on chronic kidney disease or dialysis, suffering from liver cirrhosis, or those who received a preoperative vitamin $\mathrm{K}$, fresh frozen plasma, or prothrombin complex concentrates.

\section{Data Collection and Outcome Measures}

Only subjects who met the inclusion criteria and provided signed informed consent were recruited. Patients were followed for two visits over the last week prior to their procedure. The first visit was a routine visit on the day of warfarin interruption (7-5 days prior to the day of the procedure) to record first INR reading. The patient had to attend a second visit on the same day or a day before the surgery, to get second INR reading. DNA sample collection was performed at any of the study visits, where patients were asked to provide either $4 \mathrm{~mL}$ of blood in BD Vacutainer ${ }^{\circledR}$ K2E EDTA $7.2 \mathrm{mg}$ glass collection tubes (Ref. No. 368861) or $2 \mathrm{~mL}$ of saliva sample single

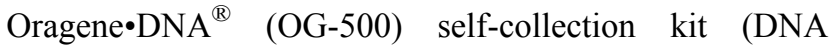
Genotek $^{\mathrm{TM}}$, Canada), according to the manufacturer's instruction.

\section{DNA Extraction and Genotyping}

Blood samples were stored in a $-20^{\circ} \mathrm{C}$ freezer until DNA isolation. Later, genomic DNA was extracted from whole blood as per the manufacturer's protocol and using PureLink $^{\circledR}$ Genomic DNA mini kits, Invitrogen ${ }^{\mathrm{TM}}$, in line with the producer standards. ${ }^{37}$ On the other hand, saliva sample kits were kept at room temperature until DNA extraction. For the DNA purification from the saliva sample, the PrepIT $^{\circledR} \cdot$ L2P standard protocol was followed. $^{38}$
The quality and quantity of the purified DNA were evaluated by Nanodrop 2000c Spectrophotometer (Thermo Fisher Scientific). ${ }^{39}$ Finally, the samples were genotyped for detecting the genetic polymorphisms in the genotypes of CYP2C $9 * 2$ (rs1799853), CYP2C $9 * 3$ (rs1057910), CYP4F $2 * 3 \quad(\mathrm{rs} 2108622), \quad$ VKORC1*2 (rs9923231), and FII (rs5896) and FVII (rs3093229) using the real-time Polymerase Chain Reaction (PCR) QuantStudio $^{\text {TM }}$. Single nucleotide polymorphism (SNP) genotyping assay probes were manufactured by Applied Biosystems $^{\mathrm{TM}}$; and their context sequences are listed in Supplementary Table 1. Demographics (age, nationality, gender, weight, height, etc.) and clinical data (concurrent medications, co-morbidities, INR target, etc.) were obtained directly from the patients and their EHR.

\section{Study Outcome}

The primary study outcome is the INR drop rate (equals to the difference between INR readings, divided by the days' interval) between carriers and non-carriers of the genetic variants.

\section{Sample Size Calculation}

The sample size for multiple regression was calculated using http://www.danielsoper.com/statcalc ${ }^{40}$ based on effect size (0.2), 0.8 statistical power, 0.05 alpha level, and 20 predictors, the required sample size was 122 patients. Counting for an anticipated $20 \%$ drop-out rate, the sample size was estimated to be 146 .

\section{Statistical Analysis}

Descriptive analysis was used to analyze genetics, demographics, and clinical information. Continuous data were presented as mean \pm SD for normally distributed data, the median and interquartile range (IQR) for non-normally distributed data. Categorical variables were described as frequencies and percentages. Independent Student's $t$-test and one-way ANOVA test were used for comparing normally distributed continuous data. In contrast, either the Mann-Whitney $U$-test or the Kruskal-Wallis test was performed to compare non-normally distributed continuous data. To adjust for multiple comparisons for the effect of genetic variants $[$ CYP $2 C$ C $* 2, \quad * 3, \quad$ VKORC $1 * 2$ (rs9923231)], Bonferroni correction was performed by dividing the $P$-value (0.05) by the number of SNPs tested (3 SNPs). Any $P$-value of 0.016 or less was considered significant. Chi-square- goodness-of-fit was used to confirm that all allele frequencies for the tested genetic variants fit the Hardy-Weinberg equilibrium (HWE). 
A $P$-value of more than 0.05 indicated that allele frequencies fit HWE. The INR decline rate (slope) was calculated as the ratio between the difference in INR values (delta INR) and the difference in days at which these INRs were measured (delta days). Single linear regression (SLR) was performed to assess the impact of genetic, clinical, and demographic factors as a continuous variable on the rate of INR decline. Multiple linear regression (MLR) modeling was used to determine the factors associated with the INR decline and to develop INR normalization model. Logistic regression was used to test the association between genetic and non-genetic factors with an INR of $\leq 1.2$ versus $>1.2$ on the day before or on the day of the planned surgery.

A two-tailed $P$-value of $<0.05$ was considered significant. IBM Statistical Package for Social Science (IBM SPSS 26 software; IBM, New York) was used to carry out the statistical analysis.

\section{Results}

\section{Patient Enrollment and Population Characteristics}

Out of 129 approached patients, 115 patients were recruited (11\% rejection rate). Additional 14 subjects were excluded due to various reasons, as illustrated in Figure 1. To expand the sample size, data of 15 eligible patients from previous warfarin research project ${ }^{20}$ was integrated into the current analysis to make the total sample size 116. These patients agreed on using their information in future research. The mean $\pm \mathrm{SD}$ age of the cohort was $66.2 \pm 14.4$ years and were almost equal proportions of both genders [65 (56\%) were men]. Table 1 shows patients' demographics and characteristics. The majority of the patients $(41.4 \%)$ were locals (Qataris) and had multiple comorbidities (mean \pm SD number of co-morbidities was $4 \pm 2.1$ ). Most of the patients $(63.8 \%)$ had hypertension; besides, half of them $(50 \%)$ were suffering from diabetes mellitus and/ or dyslipidemia. Of the drugs reported as INR and warfarin therapeutic effect inducer, 33 patients were using aspirin, clopidogrel, or both. Seven patients were taking non-steroidal anti-inflammatory drugs (NSAIDs), four were taking amiodarone, one patient was taking metronidazole and another one was on ciprofloxacin. On the other hand, of the drugs stated as a possible reducer of warfarin effect and INR, three patients were on rifampin, one patient was using carbamazepine and one was taking phenytoin.
Most of the participants were taking warfarin for the indication of stroke prevention in atrial fibrillation (AF) or mechanical/ bioprosthetic heart valve replacement [63 (54.3\%) and 47 (40.5\%), respectively]. The mean \pm SD INR reading at the time of interruption and day of procedure were 2 \pm 0.4 and $1.2 \pm 0.13$, respectively. Types of performed procedures are summarized in Supplementary Table 2.

\section{Prevalence of Genetic Variants}

MAF was computed to estimate the prevalence of genetic variants. No deviation from Hardy-Weinberg equilibrium (HWE) were detected for any genotype frequencies (Table 2). Table 3 presents the genotype frequencies.

\section{The Effect of Genetic Factors on Weekly Warfarin Maintenance Dose}

Warfarin's weekly dose ranged widely from $7.0 \mathrm{mg}$ to $91.0 \mathrm{mg} / \mathrm{week}$. As expected, the results of non-parametric analysis indicated that carriers of one or two copies of decreased or no function allele of CYP2C $\left(C Y P 2 C 9^{*} 2\right.$ or *3) required a significant lower median (IQR) weekly maintenance dose compared to non-carriers' allele $\left(* 1 /{ }^{*} 1\right)[24.7$ (21.1) $\mathrm{mg} / \mathrm{week}$ vs $35(21) \mathrm{mg} / \mathrm{week}, p=0.001)$ (Figure 2). Similarly, carriers of VKORCl $^{* 2} \quad(-1639 \mathrm{G}>\mathrm{A})$ had a significant lower median (IQR) weekly maintenance dose compared to the wild-type allele [28 (21) $\mathrm{mg} /$ week vs 42 (23.8) $\mathrm{mg} / \mathrm{week}, p<0.0001$ ) (Figure 3).

\section{The Effect of Genetic and Non-Genetic Factors on INR Decline Rate}

Since the cohort's INR decline rate (slope) was skewed, log transformation was performed before the regression analysis. All variables were tested for association with the log transformation rate of INR decline using univariate linear regression, and the following (clinical, genetic, and non-genetic) variables had a $p$-value of $<0.2$ and thus, were included in the multiple linear regression: cancer status, ciprofloxacin, antiplatelet medication, antibiotics, INR goal, and INR index (INR at visit 1). Stepwise forward selection regression model showed that ciprofloxacin, antiplatelet medications, and INR index are the only factors associated with the INR decline rate. Table 4 demonstrates that INR decline was steeper (required less time to reach baseline INR) for patients with a higher INR index or who took antiplatelet medications ( $p<0.0001$ and $p=0.014$, respectively). In contrast, it was lower (required more time to reach baseline INR) for those who were using ciprofloxacin $(p=0.001)$. 


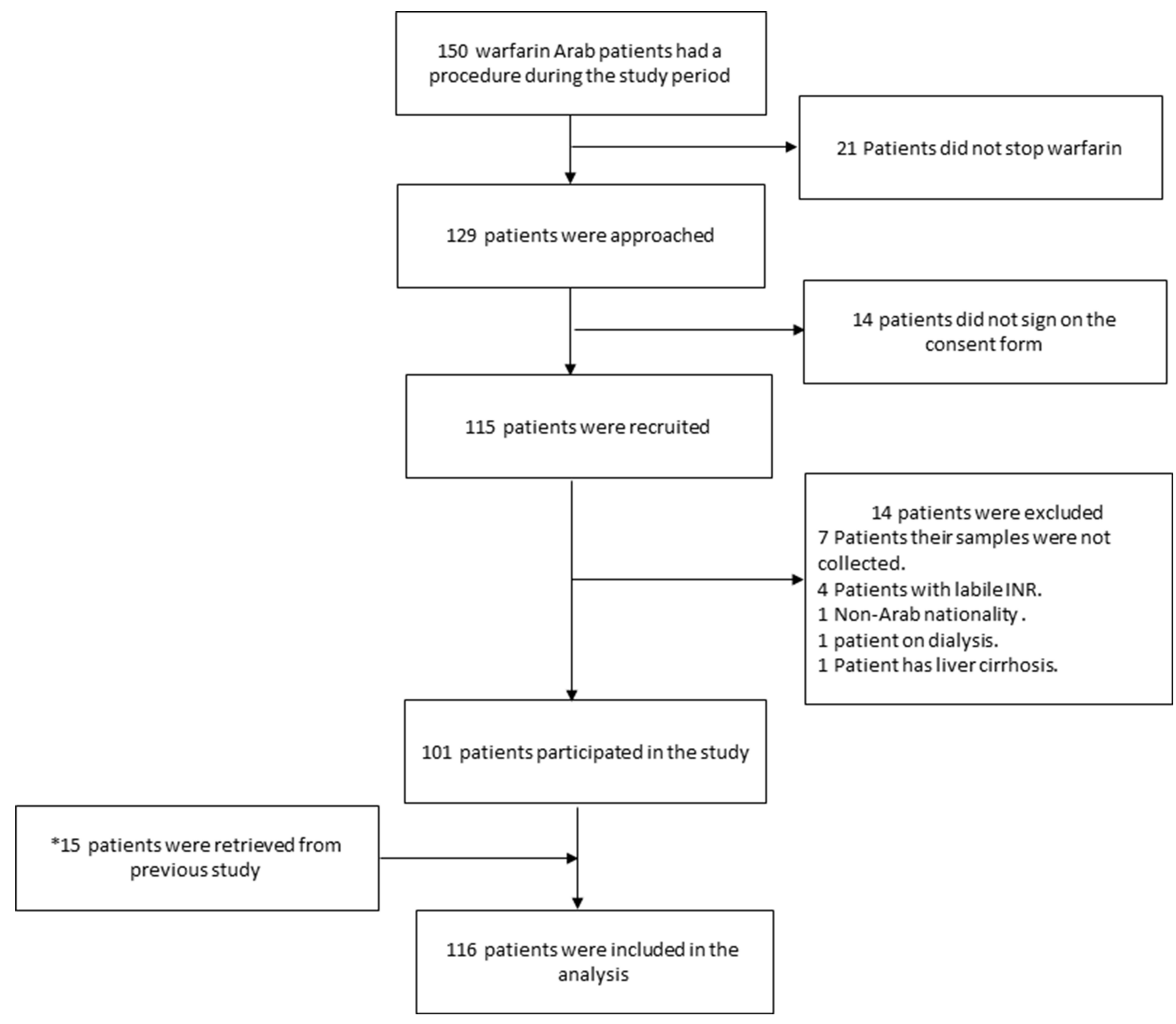

Figure I Flowcharts of patients included in the analysis. $\left(* \mathrm{~L}\right.$. Bader et $\left.\mathrm{al}^{20}\right)$.

\section{The Effect of Genetic and Non-Genetic Factors on Achieving Normalized INR $(\leq 1.2)$}

The proportion of $C Y P 2 C 9 * 3$ carriers achieving normalized INR $(\leq 1.2)$ on the day of surgery was significantly lower than the wild-type allele ( $28 \%$ vs $60 \%, p=0.013$ ) (Figure 4). Whereas the percentage of $C Y P 2 C 9 * 2$ carriers reaching normalized INR $(\leq 1.2)$ on the day of operation was not different than that of the wild-type allele ( $63 \%$ vs $60 \%, p=0.80$ ).

Variables association with achieving INR $\leq 1.2$ were tested in univariate analysis. Those with $p$-value $<0.2$ were added to the binary logistic regression model (Supplementary Table 3). Logistic regression, in both stepwise forward selection and backward elimination, proved that heparin bridging, INR target, and Sudanese nationality are significant predictors of INR normalization $(\leq 1.2)$ on the day of the procedure. The results showed that INR $\leq 1.2$ is associated with the presence of INR goal $\leq 3.5$, heparin bridging and being of any Arab nationality rather than Sudanese (Table 5). Although CYP2C $9 * 3$ genotype was a significant predictor of normalized INR in univariate analysis, it was not significantly associated in the multivariate model.

\section{Discussion}

This study was set out to identify the genetics, demographics, and clinical factors contributing to the time to normalize INR after the withdrawal of warfarin in 
Table I Patient Characteristics

\begin{tabular}{|c|c|}
\hline Characteristic & $\begin{array}{l}\text { Cohort } \\
(\mathrm{N}=1 \mid 6)\end{array}$ \\
\hline Age, in years, mean $\pm S D$ & $66.2 \pm 14.4$ \\
\hline $\mathrm{BMI}$ in $\mathrm{kg} / \mathrm{m}^{2}$, mean $\pm \mathrm{SD}$ & $30.0 \pm 5.7$ \\
\hline Male sex, no. (\%) & $65(56)$ \\
\hline Nationality, no. (\%) & \\
\hline Qatari & $48(4 \mid .4)$ \\
\hline Egyptian & $22(19.0)$ \\
\hline Palestinian & $16(13.8)$ \\
\hline Sudanese & $10(8.6)$ \\
\hline Others ${ }^{\dagger}$ & $20(17.2)$ \\
\hline Smoker, no. (\%) & $14(12.1)$ \\
\hline Amount of vitamin $\mathrm{K}$ rich food intake, no. (\%) $¥$ & \\
\hline Low & $26(22.4)$ \\
\hline Medium & $86(74.1)$ \\
\hline High & $4.0(3.4)$ \\
\hline Current medical condition, no (\%) & \\
\hline Diabetes mellitus & $58(50.0)$ \\
\hline Hypertension & $74(63.8)$ \\
\hline Congestive heart failure & $13(11.2)$ \\
\hline Cancer & $9.0(7.8)$ \\
\hline Dyslipidemia & $58(50.0)$ \\
\hline Thyroid's dysfunction ${ }^{\beta}$ & $14(12.0)$ \\
\hline Co-morbidities, mean $\pm \mathrm{SD}$ & $4.0 \pm 2.1$ \\
\hline Current interacting medications, no. (\%) & \\
\hline Amiodarone & $4.0(3.4)$ \\
\hline Metronidazole & $1.0(0.9)$ \\
\hline Ciprofloxacin & $1.0(0.9)$ \\
\hline Carbamazepine & $1.0(0.9)$ \\
\hline Rifampin & $3.0(2.6)$ \\
\hline NSAIDs & $7.0(6.0)$ \\
\hline PPIs & $66(56.9)$ \\
\hline Statins & $75(64.7)$ \\
\hline Antiplatelets & $33(28.4)$ \\
\hline Warfarin indication, no. (\%) & \\
\hline $\mathrm{AF}$ & $63(54.3)$ \\
\hline Mechanical or bioprosthetic heart valve replacement & $47(40.5)$ \\
\hline VTE & $17(14.7)$ \\
\hline Thrombophilia & $6.0(5.2)$ \\
\hline Stroke & $15(12.9)$ \\
\hline Others * & $6(5.2)$ \\
\hline INR target, no. (\%) & \\
\hline $2.0-3.0$ & $63(71.6)$ \\
\hline $2.5-3.5$ & $22(19.0)$ \\
\hline Others $^{\gamma}$ & II (9.5) \\
\hline Maintenance weekly dose, mean \pm SD & $29.8 \pm 14.0$ \\
\hline
\end{tabular}

(Continued)
Table I (Continued).

\begin{tabular}{|l|c|}
\hline Characteristic & $\begin{array}{c}\text { Cohort } \\
(\mathbf{N}=1 \text { I l6) }\end{array}$ \\
\hline Ist INR reading, mean \pm SD & $2.0 \pm 0.4$ \\
\hline 2nd INR reading, mean \pm SD & $1.2 \pm 0.13$ \\
\hline $\begin{array}{l}\text { Difference between Ist and 2nd INR readings, } \\
\text { mean } \pm \text { SD }\end{array}$ & $0.84 \pm 0.38$ \\
\hline Days of discontinuation, median (IQR) & $5.0(2.0)$ \\
\hline INR decline rate, median (IQR) & $0.45(0.56)$ \\
\hline INR <I.5 at time of procedure, no (\%) & $96(82.8)$ \\
\hline INR $\leq 1.2$ at time of procedure, no (\%) & $65(56.1)$ \\
\hline
\end{tabular}

Notes: ${ }^{\dagger}$ According to the League of the Arab States; ${ }^{\sharp}$ Amount of vitamin K-rich food intake was defined as low; I-2 bowl/week, medium; 3-4 bowls/week, high; 5-7 bowls/weeks; ${ }^{\beta}$ Thyroid dysfunction was defined as patients with hypo-or hyperthyroids; *Others was defined as some patients are taking warfarin for more than one indication; ${ }^{\gamma}$ Others is $2-2.5 / 3-3.5 / 3-4$.

Abbreviations: AF, atrial fibrillation; BMl, body mass index; INR, international normalization ratio; IQR, interquartile range; NSAIDs, non-steroidal antiinflammatory drugs; PPI, proton pump inhibitor; SD, standard deviation; VTE, venous thromboembolism.

periprocedural management in Arab patients. In 2017, Elewa et $\mathrm{al}^{2}$ reported that warfarin prescription represented $77 \%$ of total OAC use in Qatar, indicating that warfarin is still primarily used despite an increased interest in direct oral anticoagulants. Unfortunately, there is a high inter-and intra-individual variability in warfarin response. ${ }^{8}$ This variability is not seen only during warfarin dosing but also during warfarin interruption in periprocedural management. Therefore, it was reasonable to investigate the factors that may affect INR decline after warfarin discontinuation during the preprocedural time.

Previous works evaluating the relation between INR decline and CYP2C9, CYP4F2, VKORC1 genetics polymorphism observed inconsistent results. Three studies $^{30,31,33}$ declined to find any significant genetic predictor of INR decline rate because of their small sample size, which made them not powered enough to detect any difference between carriers and non-carriers of genetics polymorphism. Some of these studies were also limited by the retrospective study design and potential selection bias. ${ }^{30,31}$ Nevertheless, some reported only an impact of CYP2C9 genetic polymorphic alleles on INR normalization. ${ }^{32,34}$ Since early 2000 , it was observed that the $C Y P 2 C 9$ genetic variant is a significant predictor of the warfarin clearance as an outcome in vivo that may be sound representative of INR decline as an outcome. ${ }^{32,34}$ 
Table 2 Minor Allele Frequency of Genotypes

\begin{tabular}{|l|c|c|c|c|c|c|}
\hline Genotype & CYP2C9*2 & CYP2C9*3 & CYP4F2*3 & VKORCI*2 (-1639G>A) & FII (C>T) (rs5896) & FVII (C>T) (rs3093229) \\
\hline SNP ID & rs I799853 & rs I057910 & rs2108622 & rs992323I & rs5896 & rs3093229 \\
MAF & 0.12 & 0.08 & 0.41 & 0.46 & 0.09 & 0.09 \\
P-value* & 0.227 & 0.105 & 0.489 & 0.297 & 0.259 & 0.300 \\
\hline
\end{tabular}

Note: *If $P \geq 0.05$ - consistent with HWE.

Abbreviations: CYP2C9, cytochrome P450- family 2- subfamily C- member 9; CYP4F2, cytochrome P450- family 4- subfamily F- member 2; FII, coagulation factor II; FVII, coagulation factor VII; HWE, Hardy-Weinberg equilibrium; MAF, Minor allele frequency; SNP, single nucleotide polymorphism; VKORCI, vitamin K epoxide reductase complex subunit I.

Table 3 Frequency Distribution of Different Genotypes

\begin{tabular}{|c|c|}
\hline Genotype Frequencies No. (\%) & Cohort $(\mathrm{N}=|| 6)$ \\
\hline \multicolumn{2}{|l|}{ CYP2C9 *2 (C>T) (rsI799853) } \\
\hline $\mathrm{CC}$ & $90(77.6)$ \\
\hline $\mathrm{CT}$ & $22(19.0)$ \\
\hline TT & $4.0(34)$ \\
\hline \multicolumn{2}{|l|}{ CYP2C9 $* 3(A>C)(r s \mid 057910)$} \\
\hline AA & 98 (84.5) \\
\hline$A C$ & $16(13.8)$ \\
\hline $\mathrm{CC}$ & $2.0(1.7)$ \\
\hline \multicolumn{2}{|l|}{ CYP4F2*3 (C>T) (rs2108622) } \\
\hline $\mathrm{CC}$ & $41(35.3)$ \\
\hline $\mathrm{CT}$ & $52(44.8)$ \\
\hline $\mathrm{TT}$ & $23(19.8)$ \\
\hline \multicolumn{2}{|l|}{ VKORCI*2 -I639G>A (C>T) (rs992323I) } \\
\hline $\mathrm{CC}$ & $37(31.9)$ \\
\hline CT & $52(44.8)$ \\
\hline $\mathrm{TT}$ & $27(23.3)$ \\
\hline \multicolumn{2}{|l|}{ FIl $(C>T)(r s 5896)$} \\
\hline $\mathrm{CC}$ & $94(81.0)$ \\
\hline CT & $22(19.0)$ \\
\hline TT & $0.0(0.0)$ \\
\hline \multicolumn{2}{|l|}{ FVII (C>T) (rs3093229) } \\
\hline $\mathrm{CC}$ & $96(82.8)$ \\
\hline $\mathrm{CT}$ & $18(15.5)$ \\
\hline TT & $2.0(1.7)$ \\
\hline
\end{tabular}

Abbreviations: CYP2C9, cytochrome P450-family 2- subfamily C-member 9; CYP4F2, cytochrome P450-family 4-subfamily F-member 2; FIl, coagulation factor II; FVII, coagulation factor VII; VKORCI, vitamin $\mathrm{K}$ epoxide reductase complex subunit I.

Abohelaika and his group from the United Kingdom found CYP2C9 genetic polymorphism to be significantly associated with INR decline in Caucasians where carriers of double CYP2C9 genetic polymorphism have more chance to fail reaching INR $<1.5$ prior to the procedure compared to non-carriers (OR, 8.64, 95\% CI, 2.25-33.25). However, the study failed to find any correlation between INR decline rate and warfarin clearance. ${ }^{28,29}$ While our study did not identify any significant genetic polymorphism associated with the INR decline rate, there was a signal that $C Y P 2 C{ }^{*} 3$ carriers are less likely to achieve INR $\leq 1.2$ at the time of the procedure. This somewhat expected finding could be attributed to the strong inhibitory effect of $C Y P 2 C 9 * 3$ on the enzyme activity compared to the effect of $C Y P 2 C 9 * 2(95 \%$ vs $30-88 \%){ }^{41,42}$

Another finding is that $44 \%$ of the participants had INR $>1.2$, and $17.2 \%$ experienced INR $\geq 1.5$ on the day of surgery. A possible explanation for this might be that $72 \%$ of performed procedures were minor procedures like dental intervention or endoscopy in which healthcare providers do not prefer a major drop in INR $(\leq 1.2)$ during warfarin discontinuation; however, the presence of $17.2 \%$ of patients with INR $\geq 1.5$ could lead to increasing the cost of preoperative admission, postponing, or canceling planned procedures. This rate was even more than twice what was previously reported. ${ }^{6,7}$ In one of these two cited studies, it was found that $23 \%$ of the patients who stopped warfarin attained an INR $>1.2$ and $7 \%$ reached a preoperative INR $>1.5$, while the other revealed that $17 \%$ of the subjects got INR $>1.5$ on the day of the procedure.

The results of our study indicate that the prevalence of $C Y P 2 C 9 * 2$ and $C Y P 2 C 9 * 3$ are $(12 \%$ and $8 \%$, correspondingly). This accords with an earlier systematic review, which showed that MAF of $C Y P 2 C 9^{*} 2$ and the $C Y P 2 C 9 * 3$ in the MENA region ranging between (5$12.0 \%)$ and (4-10\%), respectively, among different nationalities in the Arab region. ${ }^{18,43}$ This prevalence is primarily similar to the one previously reported in Qataris and Egyptians, which is logical since these two nationalities represented $60 \%$ of our study population. ${ }^{44,45}$ In comparison, CYP $4 F 2 * 3$ and $V K O R C 1 * 2(-1639 \mathrm{G}>\mathrm{A})$ are $41 \%$ and $46 \%$, respectively, and they were comparable to previous studies done in Egyptians ( $42 \%$ and $46 \%$, respectively) and in Qataris (43\% and $47 \%$, respectively). ${ }^{20,45}$ Looking outside the Arab States, CYP2C ${ }^{*} 2$ and CYP2C $9 * 3$ MAF were not much different from 


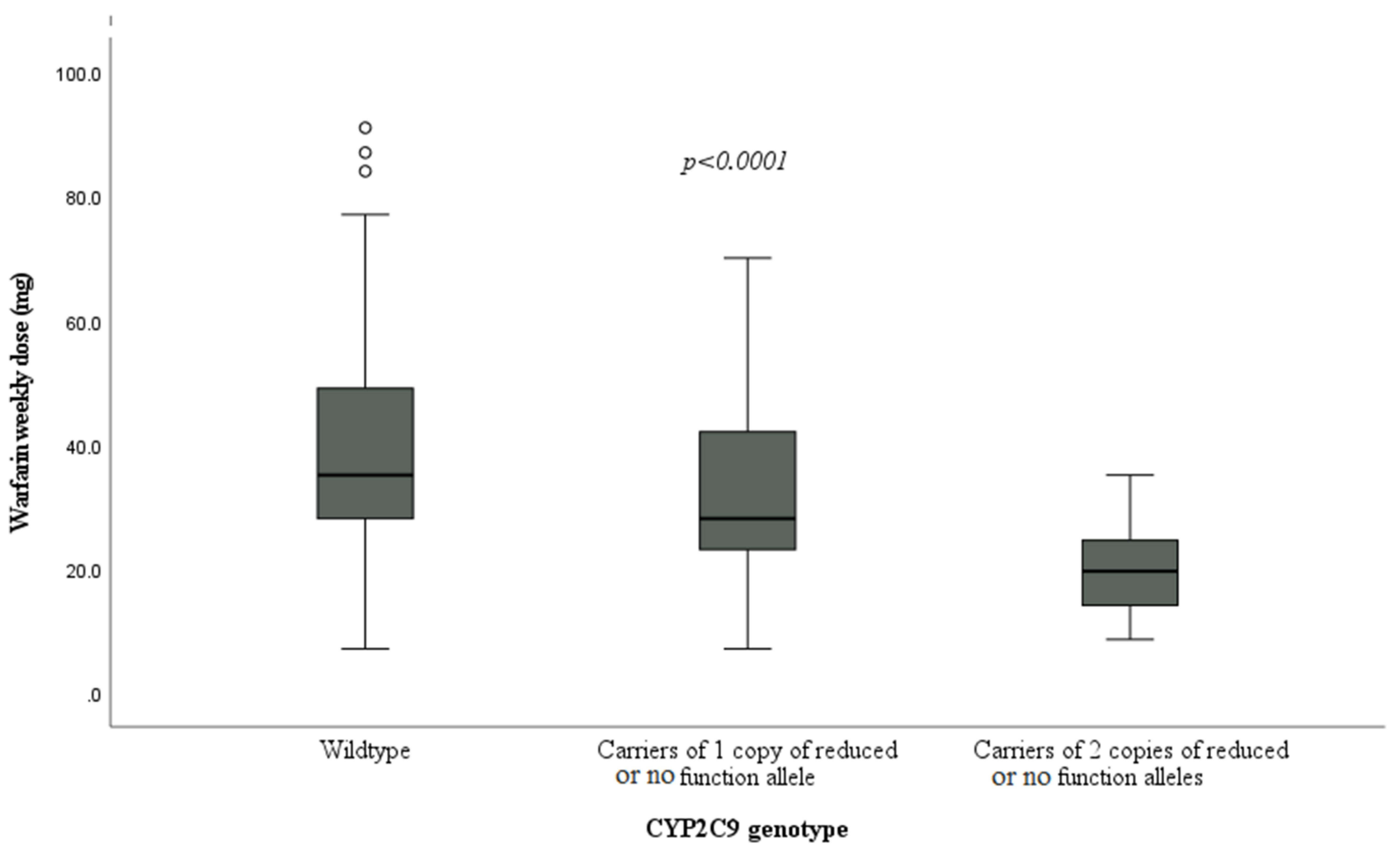

Figure 2 The effect of CYP2C9 variants allele on warfarin weekly maintenance dose. Kruskal-Wallis test was applied to compare the median warfarin dose between wildtype and carriers of $\mathrm{I}$ copy or 2 copies of reduced or no function allele. Boxes represent the median and interquartile range. Lines above and below the boxes represent maximum and minimum values.

Abbreviation: CYP2C9, cytochrome P450-family 2-subfamily C-member 9.

Europeans' prevalence (12\% and 6.6\%) and Turks (13\% and $10 \%$ ). Besides, the MAF of $C Y P 4 F 2 * 3$ was closer to South Asians and Turks (36\% and $40 \%){ }^{46}$ as well as MAF of VKORC1*2 $(-1639 \mathrm{G}>\mathrm{A})$, was equaling to Latin Americans and Turks (44\% and 49\%). ${ }^{20,47}$ The observed similarity between Arabs and Turks in the prevalence of different genotypes could be due to the long history of the Ottoman Empire's occupation, which resulted in many matings, mixing of lineages and transmission of genetic traits between Arabs and Turks. ${ }^{48}$

Our study is one of few studies that reported the variant allele frequencies for the polymorphisms of coagulation $F I I$ and FVII genetic variants. In our population, the MAF of FII $(C>T)$ (rs5896) and FVII $(C>T)$ (rs3093229) were identical (9\%). Regarding the MAF of FVII, this frequency was in line with an observation from a previous study in Jordan, which revealed that FVII's MAF was $6 \%{ }^{49}$ In contrast, this incidence was lower than other populations. ${ }^{50}$ Furthermore, the variant allele frequency of FII in our results was less than the global population and Europeans (14\%).
Another clinically relevant finding was that our results reconfirm the association of CYP2C9 and VKORC1 polymorphisms with weekly warfarin dose. It was clear that carriers of wild-type allele of CYP2C9 or VKORC1 required a higher mean warfarin dose than carriers of one or two copies of reduced or no function alleles of these genes. On the other hand, and despite its high prevalence, CYP4F2*3 did not affect warfarin dose as previously shown in studies performed on Egyptian and Qatari populations. ${ }^{20,45}$

The current study's multiple regression analysis showed that antiplatelet and ciprofloxacin use, and INR index are significant predictors of the INR decline rate. The observed negative relationship between the ciprofloxacin and INR decline rate is likely related to the suppression of vitamin K producing intestinal flora and the CYP inhibition by ciprofloxacin which leads to further delay in warfarin clearance and blunts the INR drop. ${ }^{51}$ The significant positive association between the INR index and INR decline rate means that with higher starting INR at the time of interruption, a faster drop in the rate of INR is expected. This was in agreement with the results observed by Abohelaika et $\mathrm{al}^{28}$ and Burmester et al. ${ }^{30}$ 


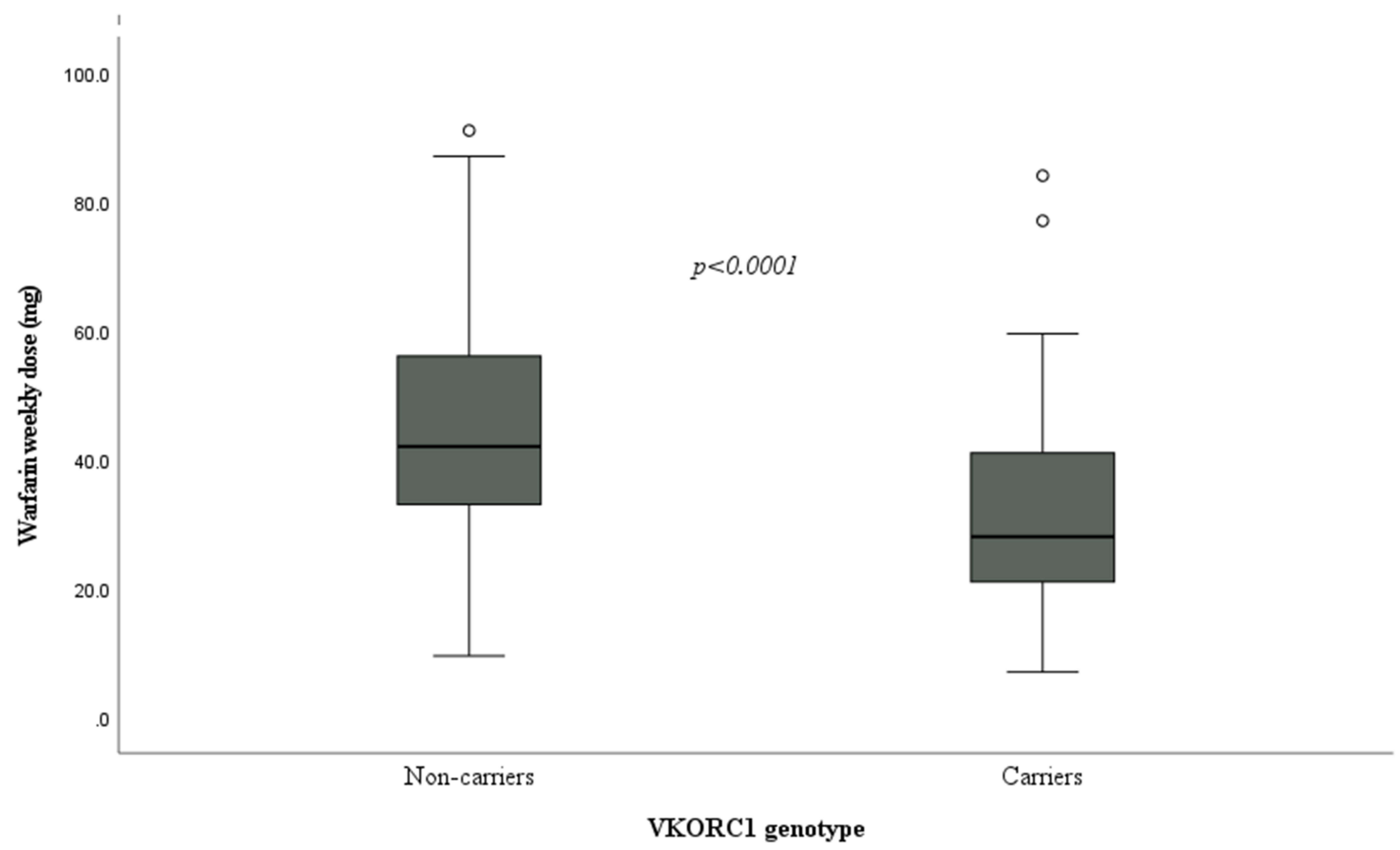

Figure 3 The effect of $V K O R C I$ variants allele on warfarin weekly maintenance dose. Mann-Whitney U-test was applied to compare the median warfarin dose between carriers and noncarriers. Boxes represent the median and interquartile range. Lines above and below the boxes represent maximum and minimum values.

Abbreviation: $V K O R C I$, vitamin $\mathrm{K}$ epoxide reductase complex subunit $\mathrm{I}$.

While the strong positive relation between antiplatelet use and the decline rate is surprising and opposite to what one would expect, it is possible that those patients interrupted the antiplatelets as well during the preprocedural period. ${ }^{52}$ Unfortunately, data on the discontinuation of antiplatelet during the periprocedural period was not collected.

There were multiple factors that showed their contribution to reaching normalized INR $\leq 1.2$. Firstly, the lower the INR target $(<3.5)$, the shorter time was to reach INR $\leq 1.2$. Secondly, being a patient of Sudanese nationality was likely to take more time to reach regular INR. The explanation of this could be that Sundanese is the only black race among the participants' races, potentially

Table 4 Multiple Linear Regression Indicating Factors Associated with INR Decline Rate

\begin{tabular}{|l|c|c|c|}
\hline Predictor & Coefficient & Standard Error & p-value* \\
\hline Antiplatelets & 0.10 & 0.04 & 0.014 \\
Ciprofloxacin & -0.66 & 0.20 & 0.001 \\
INR index & 0.31 & 0.03 & $<0.0001$ \\
\hline
\end{tabular}

Notes: ${ }^{*} p$-value $<0.05$, is significant; Model adjusted $-R^{2}=0.435, p$-value $<0.001$; ${ }^{\dagger}$ INR at first visit. carriers of other untested common SNPs in blacks like CYP 2 C $9 * 5 * 6 * 8 * 11$ which decrease CYP2C9 enzyme activity. ${ }^{53}$

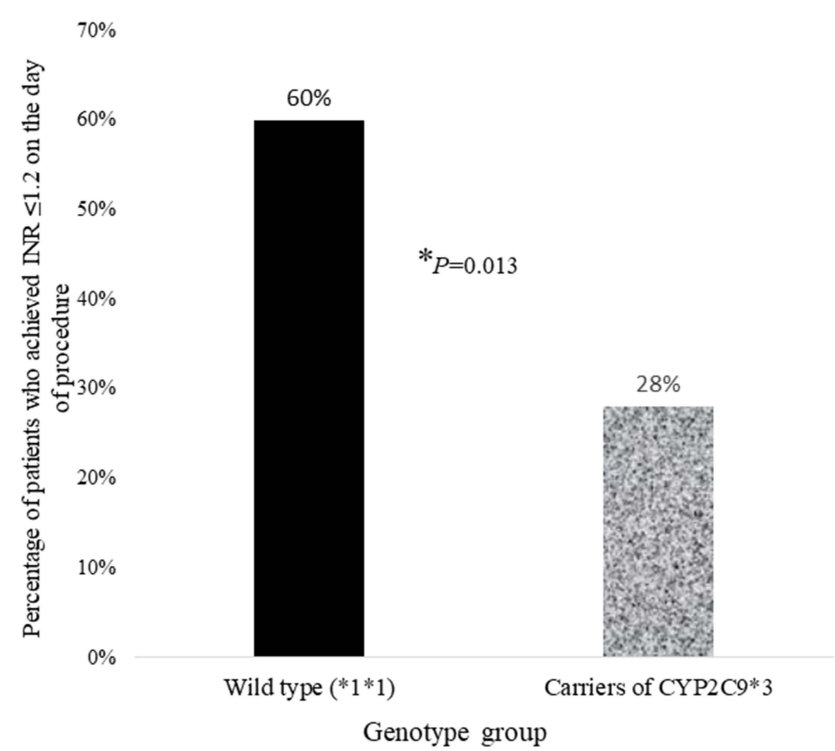

Figure 4 The proportion of individuals with CYP2C9 *3 variant alleles, their INR status before procedure. *Chi-square test was performed to compare the proportion of carrier of CYP2C9*3 with INR $\leq 1.2$ to the non-carriers of CYP2C9*3.

Abbreviations: CYP2C9, cytochrome P450-family 2-subfamily C-member 9; INR, international normalized ratio. 
Table 5 Summary Statistics and Results from Multiple Logistic Regression Analysis

\begin{tabular}{|l|c|c|c|c|c|c|}
\hline Term & Coefficient & Standard Error & Odds Ratio & P-value* & \multicolumn{2}{|c|}{$\mathbf{9 5 \%}$ Cl } \\
\cline { 3 - 7 } & & & & & & Lower \\
\hline Constant & & & & & \\
Using enoxaparin & -3.86 & 1.54 & 0.02 & 0.013 & \\
INR target $\leq 3.5$ & 2.87 & 1.12 & 13.5 & 0.020 & 1.49 & 123.33 \\
Holding a Sudanese nationality & 2.22 & 1.23 & 9.29 & 0.04 & 1.02 & 83.99 \\
\hline
\end{tabular}

Note: ${ }^{*} p$-value $<0.05$.

Abbreviations: $\mathrm{Cl}$, confidence interval; INR, international normalization ratio.

The uniqueness of this study is in its design and being one of the first studies to report the frequency of $C Y P 2 C 9$, VKORC1, CYP4F2, FII, and FVII genetic polymorphisms in Arabs. Additionally, it is the first research that investigated the effect of coagulation factors polymorphism on INR normalization. Our study has weaknesses that temper our findings. Notably, the small sample size was likely the leading cause of impeding the necessary power to detect the various significant impact of genetic variants. The small sample size was primarily due to the slow recruitment and low flow of eligible patients. Moreover, the unprecedented situation of the Coronavirus disease 2019 (COVID-19) pandemic has resulted in suspending elective surgeries for 6 months at our local clinical setting. To lighten this up, we included eligible patients from previous research. ${ }^{20}$ Another limitation is that not all participants stopped warfarin for the same period, and they had different INR targets. Nevertheless, our analysis revealed no significant difference between the status of INR $\leq 1.2$ and the INR decline rate (expressed as log slop) which considers the days of interruption and INR index. To develop a complete picture of genetics polymorphism's impact on INR decline rate, an additional more extensive study with patient stratification according to the current results will be needed to eliminate the effect of potential confounders. Equally important, cost-effective analysis of implementing pharmacogenetics-based algorithm will guide decision-makers to which approach must be subsidized.

\section{Conclusion}

This study explored the impact of genetics and non-genetic factors on INR normalization in the Arab population. Index INR and interacting medications were significant predictors of INR decline. Moreover, the study confirmed the effect of CYP2C9 and VKORC1 genetics polymorphism and their contribution to warfarin maintenance dose variability. While there was a signal that $C Y P 2 C 9 * 3$ variant may contribute to the variability in the INR decline across patients, this requires to be confirmed in future research.

\section{Ethics Approval}

Ethical approval was obtained from the Institutional Review Board (IRB) of Hamad Medical Corporation (HMC-Protocol\# MRC-16415/16) and Qatar University (QU-IRB 1296-FBA/20). This study was conducted in accordance with the Declaration of Helsinki.

\section{Acknowledgments}

The authors acknowledge clinical pharmacists' support at anticoagulation clinics; Ammar Al Abdullah, Asma Ibrahim, Dina Elgaily, Lolwa Barakat, Mehak Khalid, Mohamed Kassem, Rana M AlAdawi. The support of all nursing teams is thankfully acknowledged.

\section{Funding}

This work was supported by Hamad Medical Corporation MRC [grant number 1698/2017].

\section{Disclosure}

The authors report no conflicts of interest for this work and declared no conflicts of interest for the research, authorship, or publication of this article.

\section{References}

1. Ansell J, Hirsh J, Hylek E, Jacobson A, Crowther M, Palareti G. Pharmacology and management of the vitamin $\mathrm{K}$ antagonists: American College of Chest Physicians evidence-based clinical practice guidelines. Chest. 2008;133(6Suppl):160S-198S.

2. Elewa H, Alhaddad A, Al-Rawi S, Nounou A, Mahmoud H, Singh R. Trends in oral anticoagulant use in Qatar: a 5-year experience. J Thromb Thrombolysis. 2017;43(3):411-416. doi:10.1007/s11239017-1474-4

3. Chang C, Hoyos M, Owusu Y, Elewa H. Direct oral anticoagulant use in atypical thrombosis-related conditions. Ann Pharmacother. 2018;52 (2):185-197. doi:10.1177/1060028017731850 
4. Jones M, McEwan P, Morgan CL, Peters JR, Goodfellow J, Currie CJ. Evaluation of the pattern of treatment, level of anticoagulation control, and outcome of treatment with warfarin in patients with non-valvar atrial fibrillation: a record linkage study in a large British population. Heart. 2005;91(4):472-477. doi:10.1136/ hrt.2004.042465

5. Kim JH, Song YB, Shin DH, et al. How well does the target INR level maintain in warfarin-treated patients with non-valvular atrial fibrillation? Yonsei Med J. 2009;50(1):83-88. doi:10.3349/ ymj.2009.50.1.83

6. White RH, McKittrick T, Hutchinson R, Twitchell J. Temporary discontinuation of Warfarin therapy: changes in the international normalized ratio. Ann Intern Med. 1995;122(1):40-42. doi:10.7326/ 0003-4819-122-1-199501010-00006

7. Kovacs MJ, Kearon C, Rodger M, et al. Single-arm study of bridging therapy with low-molecular-weight heparin for patients at risk of arterial embolism who require temporary interruption of warfarin. Circulation. 2004;110(12):1658-1663. doi:10.1161/01. CIR.0000142859.77578.C9

8. Spyropoulos AC, Turpie AG, Dunn AS, et al. Clinical outcomes with unfractionated heparin or low-molecular-weight heparin as bridging therapy in patients on long-term oral anticoagulants: the REGIMEN registry. J Thromb Haemost. 2006;4(6):1246-1252. doi:10.1111/ j.1538-7836.2006.01908.x

9. Pengo V, Cucchini U, Denas G, et al. Standardized low-molecularweight heparin bridging regimen in outpatients on oral anticoagulants undergoing invasive procedure or surgery: an inception cohort management study. Circulation. 2009;119(22):2920-2927. doi:10.1161/ CIRCULATIONAHA.108.823211

10. Douketis JD, Spyropoulos AC, Spencer FA, et al. Perioperative management of antithrombotic therapy: antithrombotic therapy and prevention of thrombosis, 9th ed: American College of Chest Physicians evidence-based clinical practice guidelines. Chest. 2012;141(2Suppl):e326S-e350S. doi:10.1378/chest.11-2298

11. O'Reilly RA. Studies on the optical enantiomorphs of warfarin in man. Clin Pharmacol Ther. 1974;16(2):348-354. doi:10.1002/ cpt1974162348

12. Rettie AE, Korzekwa KR, Kunze KL, et al. Hydroxylation of warfarin by human cDNA-expressed cytochrome P-450: a role for $\mathrm{P}-4502 \mathrm{C} 9$ in the etiology of (S)-warfarin-drug interactions. Chem Res Toxicol. 1992;5(1):54-59. doi:10.1021/tx00025a009

13. Kaminsky LS, Zhang ZY. Human P450 metabolism of warfarin. Pharmacol Ther. 1997;73(1):67-74. doi:10.1016/S0163-7258(96) 00140-4

14. Garcia DA, Ageno W, Libby EN, Bibb J, Douketis J, Crowther MA. Perioperative anticoagulation for patients with mechanical heart valves: a survey of current practice. J Thromb Thrombolysis. 2004;18(3):199-203. doi:10.1007/s11239-005-0346-5

15. Stubbins MJ, Harries LW, Smith G, Tarbit MH, Wolf CR. Genetic analysis of the human cytochrome P450 CYP2C9 locus. Pharmacogenetics. 1996;6(5):429-439. doi:10.1097/00008571199610000-00007

16. Caudle KE, Dunnenberger HM, Freimuth RR, et al. Standardizing terms for clinical pharmacogenetic test results: consensus terms from the Clinical Pharmacogenetics Implementation Consortium (CPIC). Genet Med. 2017;19(2):215-223. doi:10.1038/gim.2016.87

17. Caldwell MD, Awad T, Johnson JA, et al. CYP4F2 genetic variant alters required warfarin dose. Blood. 2008;111(8):4106-4112. doi:10.1182/blood-2007-11-122010

18. Bader LA, Elewa H. The impact of genetic and non-genetic factors on warfarin dose prediction in MENA region: a systematic review. PLoS One. 2016;11(12):e0168732. doi:10.1371/journal. pone. 0168732

19. Suttie JW. The biochemical basis of warfarin therapy. Adv Exp Med Biol. 1987;214:3-16.
20. Bader L, Mahfouz A, Kasem M, et al. The effect of genetic and nongenetic factors on warfarin dose variability in Qatari population. Pharmacogenomics J. 2020;20(2):277-284. doi:10.1038/s41397-0190116-y

21. Gammal RS, Court MH, Haidar CE, et al. Clinical Pharmacogenetics Implementation Consortium (CPIC) Guideline for UGT1A1 and Atazanavir Prescribing. Clin Pharmacol Ther. 2016;99(4):363-369. doi:10.1002/cpt.269

22. Nutescu EA. Oral anticoagulant therapies: balancing the risks. $A m$ J Health Syst Pharm. 2013;70(10 Suppl 1):S3-11. doi:10.2146/ ajhp130040

23. dbSNP Short Genetic Variations; rs3093233; Factor VII; [cited July 19, 2019]. NIH; National Library of Medicine. Available from: https:// www.ncbi.nlm.nih.gov/snp/rs3093233. Accessed August 16, 2021.

24. dbSNP Short Genetic Variations; rs3093229; Factor VII; 2019 [cited July 19, 2019]. NIH; National Library of Medicine. Available from: https://www.ncbi.nlm.nih.gov/snp/rs3093229. Accessed August 16, 2021.

25. D'Ambrosio RL, D'Andrea G, Cappucci F, et al. Polymorphisms in factor II and factor VII genes modulate oral anticoagulation with warfarin. Haematologica. 2004;89(12):1510-1516.

26. Aquilante CL, Langaee TY, Lopez LM, et al. Influence of coagulation factor, vitamin $\mathrm{K}$ epoxide reductase complex subunit 1, and cytochrome P450 2C9 gene polymorphisms on warfarin dose requirements. Clin Pharmacol Ther. 2006;79(4):291-302. doi:10.1016/j.clpt.2005.11.011

27. Schelleman H, Chen J, Chen Z, et al. Dosing algorithms to predict warfarin maintenance dose in Caucasians and African Americans. Clin Pharmacol Ther. 2008;84(3):332-339. doi:10.1038/clpt.2008.101

28. Abohelaika S, Wynne H, Avery P, Kamali F. Influence of CYP2C9 polymorphism on the fall in international normalized ratio in patients interrupting warfarin therapy before elective surgery. J Thromb Haemost. 2015;13(8):1436-1440. doi:10.1111/jth.13014

29. Abohelaika S, Wynne H, Avery P, Kampouraki E, Kamali F. Effect of genetic and patient factors on warfarin pharmacodynamics following warfarin withdrawal: implications for patients undergoing surgery. Thromb Res. 2018;171:167-170. doi:10.1016/j.thromres.2018.09.064

30. Burmester JK, Berg RL, Schmelzer JR, Mazza JJ, Yale SH. Factors that affect rate of INR decline after warfarin discontinuation. WMJ. 2015;114(1):16-20.

31. Chartrungsan A, Laksanabunsong P, Nimmannit A, et al. Comparison of temporary interruption of Warfarin therapy for 3 and 5 days before surgery in Thailand: a randomized controlled trial. Siriraj Med J. 2017;65(3):69-72.

32. Herman D, Locatelli I, Grabnar I, et al. Influence of CYP2C9 polymorphisms, demographic factors and concomitant drug therapy on warfarin metabolism and maintenance dose. Pharmacogenomics J. 2005;5(3):193-202. doi:10.1038/sj.tpj.6500308

33. Kadian-Dodov DL, van der Zee SA, Scott SA, et al. Warfarin pharmacogenetics: a controlled dose-response study in healthy subjects. Vasc Med. 2013;18(5):290-297. doi:10.1177/1358863X13503193

34. Takahashi H, Kashima T, Nomizo Y, et al. Metabolism of warfarin enantiomers in Japanese patients with heart disease having different CYP2C9 and CYP2C19 genotypes. Clin Pharmacol Ther. 1998;63 (5):519-528. doi:10.1016/S0009-9236(98)90103-5

35. States LotA. Members of league of the Arab States; [cited February 13, 2021]. Available from: http://www.leagueofarabstates.net/ar/abou tlas/Pages/CountryData.aspx. Accessed August 16, 2021.

36. Wilson SJ, Wells PS, Kovacs MJ, et al. Comparing the quality of oral anticoagulant management by anticoagulation clinics and by family physicians: a randomized controlled trial. Cmaj. 2003;169 (4):293-298.

37. Invitrogen. PureLink ${ }^{\circledR}$ genomic DNA kits for purification of genomic DNA; [cited October 17, 2019]. Available from: https://tools.thermo fisher.com/content/sfs/manuals/purelink_genomic_man.pdf. Accessed August 16, 2021. 
38. Genotek D. Laboratory protocol for manual purification of DNA from $0.5 \mathrm{~mL}$ of sample; [cited October 17, 2019]. Available from: http://www.dnagenotek.com/US/pdf/PD-PR-006.pdf. Accessed August 16, 2021.

39. Scientific TF. NanoDrop 2000/2000c spectrophotometer V1.0 user manual. 2021:3.

40. Soper DS. A-priori sample size calculator for multiple regression; 2019 [cited July 10, 2019]. Available from: http://www.danielsoper. com/statcalc. Accessed August 16, 2021.

41. Rettie AE, Wienkers LC, Gonzalez FJ, Trager WF, Korzekwa KR. Impaired (S)-warfarin metabolism catalysed by the R144C allelic variant of CYP2C9. Pharmacogenetics. 1994;4(1):39-42. doi:10.1097/00008571-199402000-00005

42. Linder MW. Genetic mechanisms for hypersensitivity and resistance to the anticoagulant Warfarin. Clin Chim Acta. 2001;308(1-2):9-15. doi:10.1016/S0009-8981(01)00420-X

43. dbSNP. dbSNP short genetic variations; 2020 [cited February 27, 2021]. Available from: https://www.ncbi.nlm.nih.gov/snp/ rs1799853\#frequency tab. Accessed August 16, 2021.

44. Sivadas A, Sharma P, Scaria V. Landscape of warfarin and clopidogrel pharmacogenetic variants in Qatari population from whole exome datasets. Pharmacogenomics. 2016;17(17):1891-1901. doi:10.2217/pgs-2016-0130

45. Shahin MH, Khalifa SI, Gong Y, et al. Genetic and nongenetic factors associated with warfarin dose requirements in Egyptian patients. Pharmacogenet Genomics. 2011;21(3):130-135. doi:10.1097/ FPC.0b013e3283436b86

46. dbSNP. CYP4F2 (rs2108622); 2020 [cited February 27, 2021]. Available from: https://www.ncbi.nlm.nih.gov/snp/rs2108622\#fre quency_tab. Accessed August 16, 2021.
47. dbSNP. VKORC1 (rs9923231); 2021 [cited February 27, 2021]. Available from: https://www.ncbi.nlm.nih.gov/snp/rs9923231\#fre quency_tab. Accessed August 16, 2021.

48. Mazza R. Occupation during and after the war (Middle East) encyclopedia: encyclopedia; 2017 [cited February 27, 2021]. Available from: https://encyclopedia.1914-1918-online.net/article/occupation during and after the war_middle_east. Accessed August 16, 2021.

49. Al-Eitan LN, Almasri AY, Al-Habahbeh SO. Effects of coagulation factor VII polymorphisms on warfarin sensitivity and responsiveness in Jordanian cardiovascular patients during the initiation and maintenance phases of warfarin therapy. Pharmgenomics Pers Med. 2019;12:1-8.

50. dbSNP. FVII (rs3093229): ncbi; 2021 [cited February 27, 2021]. Available from: https://www.ncbi.nlm.nih.gov/snp/rs3093229\#fre quency_tab. Accessed August 16, 2021.

51. Holbrook AM, Pereira JA, Labiris R, et al. Systematic overview of warfarin and its drug and food interactions. Arch Intern Med. 2005;165(10):1095-1106. doi:10.1001/archinte.165.10.1095

52. Shehab N, Sperling LS, Kegler SR, Budnitz DS. National estimates of emergency department visits for hemorrhage-related adverse events from clopidogrel plus aspirin and from warfarin. Arch Intern Med. 2010;170(21):1926-1933. doi:10.1001/archinternmed.2010.407

53. Shrif NE, Won HH, Lee ST, et al. Evaluation of the effects of VKORC1 polymorphisms and haplotypes, CYP2C9 genotypes, and clinical factors on warfarin response in Sudanese patients. Eur J Clin Pharmacol. 2011;67(11):1119-1130. doi:10.1007/s00228-011-1060-1
Pharmacogenomics and Personalized Medicine

\section{Publish your work in this journal}

Pharmacogenomics and Personalized Medicine is an international, peer-reviewed, open access journal characterizing the influence of genotype on pharmacology leading to the development of personalized treatment programs and individualized drug selection for improved safety, efficacy and sustainability. This journal is indexed

\section{Dovepress}

on the American Chemical Society's Chemical Abstracts Service (CAS). The manuscript management system is completely online and includes a very quick and fair peer-review system, which is all easy to use. Visit http://www.dovepress.com/testimonials.php to read real quotes from published authors. 